\title{
Combining electrodeposition and optical microscopy for probing size-dependent single nanoparticle electrochemistry
}

\author{
Jean-François Lemineur, ${ }^{[a]}$ Jean-Marc Noël, ${ }^{[a]}$ Dominique Ausserrée, ${ }^{[b]}$ Catherine Combellas, ${ }^{[a]}$ Frédéric \\ Kanoufi; $^{\star[a]}$
}

Abstract: Electrodeposition of nanoparticles, NPs, is a promising route for the preparation of highly electroactive nanostructured electrodes. By taking advantage of progressive electrodeposition, disordered arrays of wide size distribution Ag NPs are produced. Assorted with surface reaction monitored by the highly sensitive backside absorbing layer optical microscopy (BALM), such array offers a platform for screening size-dependent electrochemistry at the single NP level. Particularly, it allows rationalizing the electrodeposition dynamics at the single $>10 \mathrm{~nm} \mathrm{NP}$, up to the point of quantifying the presence of metal nanoclusters $(<2 \mathrm{~nm})$, and probing easier NP oxidation with size decrease, either through electrochemical or galvanic reaction.

Nanostructured electrodes are attracting increasing interest in the current energy challenges through the electrocatalysis of chemical reactions. In addition to properties depending on their bulk or surface chemical composition, the activity of NPs relies on their shape, size and also on inter-NP separation distances that control catalysis efficiency and product distribution. ${ }^{1}$ To reach the ideal sustainable and electrocatalytic electrode, a rational dispersion of efficient nanocatalysts onto poorly active support is needed. It can be provided through electrodeposition ${ }^{2}$ which has several advantages as control over nanoscale composition, structure diversity, and good electrical connection. We suggest that electrodeposition, assorted with appropriate in operando monitoring, can also inspect structure-related effects in NP electrochemistry. Taking advantage of progressive electrodeposition, ${ }^{2 b}$ arrays of wide size distribution of NPs were produced, which, engaged into further (electro)chemical reactions, will decipher size-related phenomena. This requires monitoring in situ and in real time the deposition process and the individual behavior of many structurally different NPs. It is afforded by optical microscopies through wide field $\left(>50 \mu \mathrm{m}^{2}\right)$, high throughput, and nanoscale resolution imaging. They also carry quantitative information over NP structure (size, composition) or to their electrochemical, EC, activity such as double layer charging, catalysis and dissolution kinetics. ${ }^{3}$

We propose to use a recently described nanoscale optical microscopy technique, BALM (backside absorbing layer microscopy), ${ }^{4}$ to quantify in situ EC processes related to the case study of Ag NPs. It is first used to explore their electrodeposition. Indeed, several in situ explorations at the single NP level ${ }^{5}$ have challenged the existing models relying on EC transients; ${ }^{6}$ they advocate for the continuous generation of

[a] J.-F. Lemineur, J.-M. Noël, C. Combellas, F. Kanouf Université Sorbonne Paris Cité, Université Paris Diderot, ITODYS, CNRS UMR 7086, 15 rue J. de Baïf, F-75013 Paris, France E-mail: Frederic.kanoufi@univ-paris-diderot.fr

[b] D. Ausserré

Maine University, Institut des Matériaux et Molécules du Mans, CNRS UMR 6283, Avenue O. Messiaen, F-72000 Le Mans, France

Supporting information for this article is given via a link at the end of the document. nanoclusters (NCs, size <2nm). These NCs are important building blocks of larger (>10nm) NPs, ${ }^{6,7}$ through diffusion and then aggregation at nuclei sites. These NCs were imaged in situ by TEM, ${ }^{5 \mathrm{C}}$ or scanning probe microscopies, ${ }^{5 \mathrm{a}, \mathrm{d}}$ while their generation and transport were dynamically monitored from EC transient in a nano-EC-cell confined to few nucleation sites, ${ }^{5 e}$ or in extremely diluted solutions. ${ }^{7}$

We propose to address by BALM the real-time (operando) widefield imaging of the electrodeposition process, at the single NP level over a wide distribution of sizes, up to the point of probing the presence of NCs. Then, the arrays of electrodeposited Ag NPs are used to inspect size-related phenomena in their oxidation engaged either electrochemically or by electrochemical Ostwald ripening.

First the sensitivity of BALM is appreciated in reference to earlier dark-field or SPR opto-electrochemical studies which inspected for Ag NPs, larger than 30nm, either their i) electrodeposition, ${ }^{3 \mathrm{C}}$ ii) dissolution ${ }^{3 \mathrm{~d}, \mathrm{e}}$ or iii) galvanic transformation into Au NPs. ${ }^{3 f}$ To detect smaller NPs, without recourse to deleterious power, a strategy consists in imaging through antireflective substrates. ${ }^{4,8}$ For a metal, pseudo-antireflective conditions are met when it is deposited as an ultrathin layer (5nm for $\mathrm{Au}$ ) on glass. ${ }^{4}$ When illuminated from the backside by an inverted microscope (Figure 1a), such BALM substrate reflects less than $1 \%$ of the incident light toward a CCD camera. As SPR or other related reflectancebased methods, ${ }^{9}$ sensitive to local refractive index (n) variations, BALM images a wide range of materials, from dielectric (Figure $1 b)$ to metal colloids (Figure 1c).

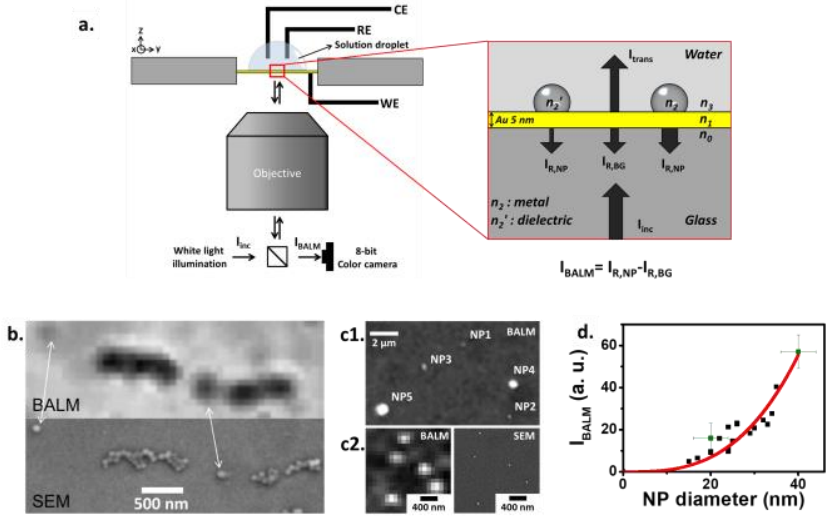

Figure 1. a) Principle of BALM imaging in water based on light reflectance contrasts; $I_{B A L M}$ is estimated from the difference at each pixel of the reflected light intensity, $I_{R}$, compared to that reflected by the substrate in the absence of $\mathrm{NP}, I_{\mathrm{R}, \mathrm{BG}}$. BALM and SEM images in the same location of b) $90 \mathrm{~nm}$ polystyrene, c1) 20 to $100 \mathrm{~nm} \mathrm{Ag}$, colloids adsorbed or c2) Ag NPs electrodeposited on a BALM substrate and detected as b) negative or c) positive contrasts. d) Sizing of (green, with error bars related to I IALM and size dispersion, averaged over $>10$ NPs) adsorbed and (black) electrodeposited Ag NPs comparing $I_{B A L M}$ and the NP size estimated from SEM; red line from Equation (1).

To a first approximation, the BALM intensity, IBALM, was rationalized from the predicted reflectance (Fresnel equations 
detailed in the Supporting Information, section S1) of 2 layers (Au thin layer and the probed material) sandwiched between a glass slide and an ambient (water) medium. This predicts, as observed in Figures 1b,c, that metal NPs are detected as bright regions compared to the substrate (increase reflectance), and conversely, dielectric NPs as darker regions (decrease reflectance). As in other optical methods relying on reflectance change, ${ }^{8,9}$ BALM senses the local change in optical thickness or optical mass (local change in the refractive index and thickness or mass). For deposited thickness values that are small compared to the wavelength, the reflectance change is generally proportional to the amount of deposited material. This lays the foundations for quantitative sizing (mass balance) during the single-NP (electro)chemical transformation.

Experimentally, the sizing performance was evaluated from images of Ag NPs, either adsorbed colloids, or electrodeposited (Figure 1d) on the Au layer. Each kind of NP is definitely differentiated in intensity, the larger the NP the brighter it is (higher $I_{B A L M}$ ). For both experiments, the background-subtracted $I_{\text {BALM }}$, for individual NPs varies linearly with the NP volume $\left(V_{\mathrm{NP}}\right)$ or as the cube of its diameter, $d_{N P}$, as in other reflectance-based methods: $:^{8,9}$

$I_{B A L M}=\alpha V_{N P}=\alpha \frac{\pi}{6}\left(d_{N P}\right)^{3}$

The correlation factor, $\alpha=1.7 \times 10^{-3} \mathrm{~nm}^{-3}$ from Figure $1 \mathrm{~d}$, depends on the Au thickness and optical noise. A reliable NP sizing requires either ex situ SEM or the pre-adsorption of a few colloidal Ag NP as internal size standards for calibration. Even with a standard 8-bit camera, NPs as small as $10 \pm 1.5 \mathrm{~nm}$ (Figure S2a, Supporting Information) were detected at the Au-water interface, making BALM over 30 -fold more sensitive (in $V_{\mathrm{NP}}$ ) than previous opto-electrochemical reports. The $I_{\text {BALM-volume }}$ (or mass) proportionality is confirmed by the reflectance model, which yields sensitivity estimates for other NPs, $15 \mathrm{~nm}$ for Pt or $\mathrm{Au}$ and $30 \mathrm{~nm}$ for polymer.

The electrodeposition and the stripping of Ag NPs at a BALM substrate were performed by sweeping its potential $(E)$ along a cyclic voltammetry (CV) in a $100 \mu \mathrm{M} \mathrm{AgNO}_{3}$ and $0.1 \mathrm{M} \mathrm{\textrm {NaNO } _ { 3 }}$ aqueous solution (no chloride contamination is expected, see section S1.2, Supporting Information). ${ }^{10}$ These processes are imaged at an acquisition rate of $20 \mathrm{~Hz}$, to dynamically size, count, and localize NPs (Figure 2a). The electrodeposition produced Ag NPs with sizes from 10 to $80 \mathrm{~nm}$, analyzed on a same image and acquisition conditions (see section S2.2, Supporting Information). Individual NP growth/stripping dynamics are inferred from the variation of $I_{\text {BALM }}$ with time. From the $I_{B A L M}$-size relationship [Equation (1)] and Faraday's law, an equivalent current, $i_{\text {opt }}$ (optical voltammogram, opCV) associated with the growth (resp. stripping) of individual NPs is inferred:

$i_{\text {opt }}=-\frac{F}{V_{m}} \frac{d V_{N P}}{d t}=-\frac{F}{\alpha V_{m}} \frac{d I_{B A L M}}{d t}$

where $V_{m}=10.27 \mathrm{~cm}^{3} \mathrm{~mol}^{-1}$ is the molar volume of $\mathrm{Ag}$ and $F$ the Faraday constant. Figure $2 \mathrm{~b}$ (and section S3, Supporting Information) shows the opCV of the electrodeposition of a $21 \pm 2 n m$ NP on the cathodic scan, and its stripping on the reverse anodic scan. Each cathodic or anodic process is characterized by a peak potential, $E_{p c}$ and $E_{p a}$, respectively. The BALM sizing ability allows relating these $E_{p}$ s to the size of the electrodeposited NPs (Figure 2d,e). Both $E_{p c}$ and $E_{p a}$ reveal a shift to more cathodic values when $d_{N P}$ decreases, with a larger shift for the electrodeposition than for the stripping, suggesting different physical origins.

Apprehending the electrodeposition dynamics allows the control of the NP size distribution. Even if nucleation is often a potentialdependent phenomenon, which leads to more complex nucleation laws, ${ }^{11}$ the evolution of the surface density of NPs, $N(t)$, along the cathodic scan of the CV (Figure 2f) can be rationalized by a potential-independent progressive nucleation law [Equation (3)]:

$N(t)=N_{0}\left(1-e^{-A \times\left(t-t_{0}\right)}\right)$

where $N_{0}$ is the NP saturation density, $A$ an apparent potentialindependent nucleation rate constant, and $t_{0}$ the induction time (or $E$ ) of the deposition. The good fit of the experimental $N(t)$ value by Equation (3) for 3s (equivalently $0.3 \mathrm{~V}$ larger than the $0.15 \mathrm{~V}$ range of $E_{p c}$ variations) suggests that the nucleation process can be analyzed by time or potential-independent $N_{0}$ and $A$ values.
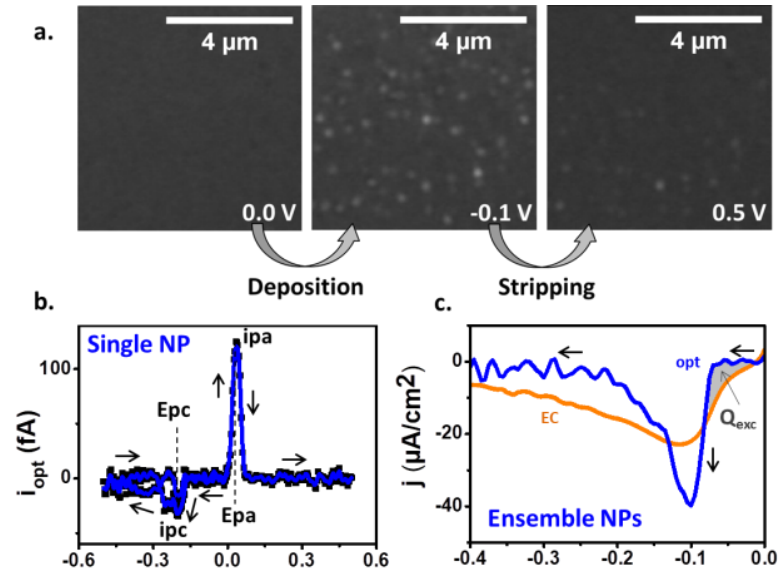

d. E (V vs AgQRE)
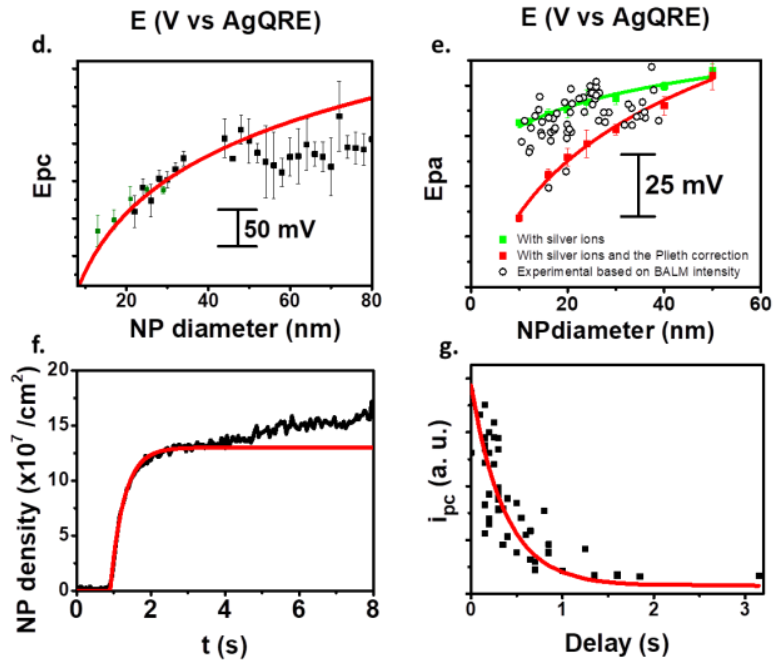

Figure 2. Monitoring the CV electrodeposition/stripping of Ag NPs (scan rate, $v=0.1 \mathrm{~V} / \mathrm{s}$ ). From a) BALM images, the variation of $I_{B A L M}$ of a bright spot (NP) is converted into b) single NP opCV (b), from which $E_{p c}$ and $E_{p a}$ size-dependency is inferred ((d) and (e), respectively). c) Comparison of background subtracted $\mathrm{EC}$ (orange) and ensemble optical (blue) current densities for $\mathrm{Ag}^{+}$reduction; the shaded area reveals the EC contribution of NCs (invisible in BALM). d,e) Ep variations with NP size, from opCVs (symbols, two independent experiments in (d), $350 \mathrm{NPs}$, binned by $3 \mathrm{~nm}$ ) compared to (d) Equation 6 or 
(e) COMSOL simulations (lines). f) NPs count over a $10 \times 10 \mu \mathrm{m}^{2}$ area during

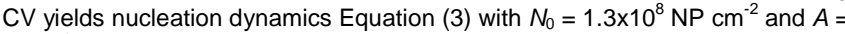
$2.7 \mathrm{~s}^{-1}$ (red = fit to equation); g) Evolution of individual NP peak currents with its birth time is fitted by Equation 5 (red line) with the same A value.

Figures 2d,g show that these expressions are satisfied experimentally with the same value of $A$ extracted from the NP count (Figure 2f), showing the full consistency of the analysis. Particularly, Equation (6) explains the observed cathodic shift of $E_{p c}$ with NP size during electrodeposition. Indeed, the $E_{p c}$ values reflect each NP birth time: the later a NP nucleates, the smaller it ends up. Unlike stated in previous report, ${ }^{3 c}$ the main contribution of the $E_{p c}$-size variations cannot be accounted for size-dependent thermodynamics (see below) but to the probability of nucleation. Equation 6 also allows controlling or inferring NP size-distribution from the nucleation rate, which can be tuned, for example, by diluting the $\mathrm{Ag}^{+}$precursor ions or examining the early stage of the nucleation.

In this early stage, NCs are generated that later aggregate into larger NPs. NCs cannot be resolved but are indirectly probed by BALM. Figure 2c compares the optical current density (blue) accumulated over $100 \mathrm{NPs}$, and the background-subtracted EC current density (orange) recorded by the potentiostat. The optical trace is delayed by $0.07 \mathrm{~V}(0.7 \mathrm{~s})$, but decreases faster and reaches a more negative peak value compared to the EC one. These discrepancies suggest a charge mismatch at the early stage of the electrodeposition, attributed ${ }^{5}$ to the NCs generation. The EC current probes the NCs electrogeneration while the opCV probes their later aggregation into NPs. The NCs density at the onset of the opCV was estimated from the EC charge density exchanged till this onset, represented by the shaded area in Figure 2c. Assuming the generation of a $2 \mathrm{~nm} \mathrm{NC}$ requires $Q_{N C}=0.04 \mathrm{fC}$, the excess charge density $Q_{e x c}=$ $3.6 \mu \mathrm{C} / \mathrm{cm}^{2}$ yields a NC density at the onset of the opCV of $\mathrm{N}_{0, N C}$ $=9 \times 10^{10} \mathrm{~cm}^{-2}$, in good agreement with reported values. ${ }^{5 \mathrm{c}}$ The NCs diffusion-aggregation should be deduced by comparing the EC and optical currents as they describe resp. the flux of NC generation and of their diffusion-aggregation into NPs, respectively, however this remains speculative owing to the small fraction of electrode optically monitored.

Once electrogenerated, the oxidation of individual NPs was also studied by BALM. When the NPs size decreases, their free energy is expected to increase as a result of an increase of surface curvature. Plieth ${ }^{12}$ proposed that the standard potential of NPs, $E_{N P}^{0}$, varies with $d_{N P}$ [Equation (7)]:

$E_{N P}^{0}=E_{b k}^{0}-\frac{4 V_{m} \gamma}{F d_{N P}}$

where $\gamma$ is the surface tension of the material, and tends toward the bulk material value, $E_{b k}^{0}$, for large NPs. For bulk $\mathrm{Ag},{ }^{13} \gamma_{\mathrm{Ag}} \approx$ 1.5 $\mathrm{Jm}^{-2}$ while Zamborini reported higher values from ensemble EC data. ${ }^{13 \mathrm{~b}}$ This shows the difficulty in properly elucidating sizedependent thermodynamics. Indeed, rather than $E_{N P}^{0}$, one measures a peak potential, $E_{p a}$, representing the behavior of the ensemble NPs. But $E_{p a}$ strongly depends on the NP surface coverage, ${ }^{13,14}$ or on possible contamination (i.e. $\mathrm{Cl}^{-}$in the sub $\mu \mathrm{M}$ range affects the $\mathrm{Ag} E_{p a}$ ). The rationalization of the electrodeposition process, presented earlier, allows generating an array of Ag NPs with a wide size distribution. Monitoring the array oxidation by BALM ensures that $E_{p a}$ for the distribution of
NPs is evaluated individually and all at once within the same experimental conditions. The local surface occupied by each NP is also monitored and accounted for, spanning from 0.3 to 1.4 $\mu \mathrm{m}^{2}$ during the stripping. The stripping within the range of NP surface coverage and sizes was simulated by COMSOL (green line in Figure 2e), showing that in the absence of Plieth correction (7), $\mathrm{E}_{\mathrm{pa}}$ remains constant within 5-10mV. Particularly, performing the stripping in the presence of $100 \mu \mathrm{M} \mathrm{Ag}^{+}$, acting as a buffer of concentration polarization, reduces (Figure S4, Supporting Information) the surface coverage impact on $E_{p a}$ and the apparent decrease of $E_{p a}$ with NP size. These effects may have been underestimated in earlier works explaining overestimation of the size-effect. ${ }^{13 b}$ Considering the Plieth correction, the simulation (red line) predicts a negative shift of $E_{p a}$ by $40 \mathrm{mV}$ when the NP decreases from 50 to $12 \mathrm{~nm}$. Experimentally (Figure $2 e$ ), the $E_{p a}$ variations suggest a $\gamma_{\mathrm{Ag}}$ value smaller than and consistent with that of bulk Ag. It confirms a recent $\mathrm{EC}$ reexamination ${ }^{13 a}$ and highlights that size-effect in $E_{N P}^{0}$ is critical to estimate for NPs larger than $10 \mathrm{~nm}$.

Finally, the sensitivity of the BALM is used to monitor the consequence of Plieth correction in a chemical environment (no polarization). Indeed, Equation (7) suggests that conductive substrates structured with Ag NPs of different sizes are unstable. Even in the absence of external polarization, when electrically contacted, a small and neighboring large NP create a Galvani corrosion cell: the large NP growing at the expense of the small one. Such a situation, known as the electrochemical Ostwald ripening, is monitored in Figure 3 but cannot be quantified from direct EC measurement as no overall current is exchanged with the exterior. Optical microscopies are able to detail its dynamic, as reported for Galvanic replacement ${ }^{3 f}$ or bipolar electrochemical systems. ${ }^{15}$

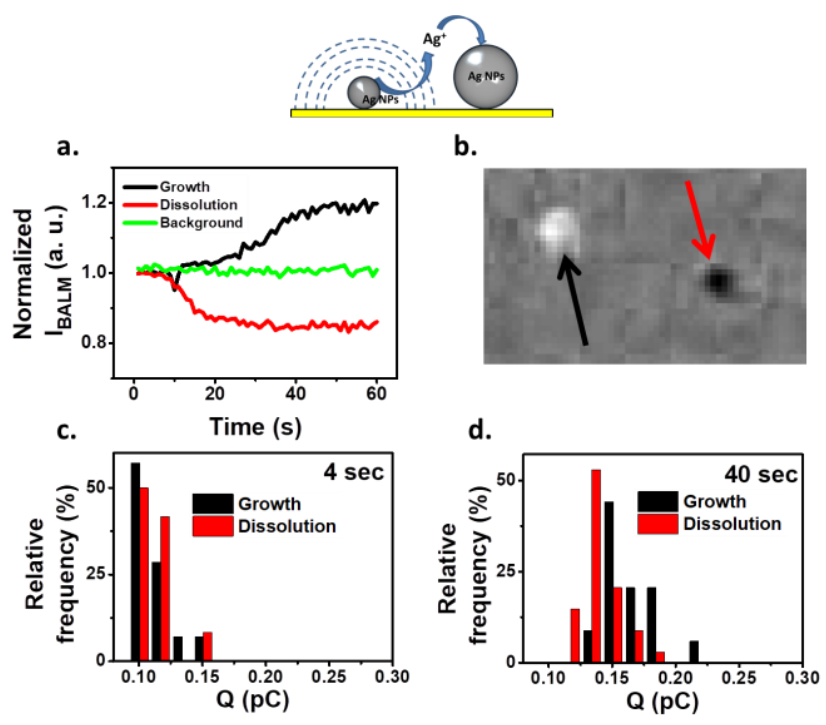

Figure 3. a) Example of normalized $I_{B A L M}$ variations of two Ag NPs over time in ultra-pure water (open circuit). b) Difference (3.1 $\mathrm{m}$ wide) between two optical images recorded at $1 \mathrm{~min}$ interval in a region where a NP dissolves (red arrow) and another grows. Charge distribution for dissolution/growth of individual NPs, calculated from BALM images, for c) 4 and d) 40 s. 
An array of electrodeposited Ag NPs of various sizes was washed and exposed to ultra-pure water, while being monitored by BALM. The I IALM transients at various locations (NPS or background) were analyzed: normalized from the $I_{B A L M}$ values at the same location on the first image they reflect local Ag material deposition/dissolution. Figure $3 a$ presents the size variations of two NPs separated by less than $2 \mu \mathrm{m}$ while Figure $3 \mathrm{~b}$ highlights them by the opposite contrasts in a difference image. Obviously, one NP grows while the other dissolves, a behavior confirmed for more than $30 \mathrm{NPs}$. Based on Equation (2), the charge transferred from each single NP growth or dissolution is estimated (for $\mathrm{t}=4$ and 40 s see Figures $3 \mathrm{c}$ and $3 \mathrm{~d}$, resp. and Figure S5a, Supporting Information). At its earliest stages, the partial or complete dissolution corresponds to $0.1 \mathrm{pC}$ (14nm NP). Meanwhile, a similar cathodic charge is detected for the NP growth suggesting the full conversion of the process and therefore a concerted mechanism. A deeper mechanistic analysis of $\mathrm{Ag}^{+}$dilution is intricate (COMSOL simulation in section S5.2, Supporting Information) as it depends on the contributions of all neighboring NPs. Such a simulation however explains that (Figure 3a) the onset of the neighbor NP growth is concomitant, but with slower rate, to the NP dissolution.

The surface reconstruction process is slow: the charges exchanged increase with time, but still with overall charge balance in agreement with a corrosion mechanism.

In conclusion BALM, which is an ultra-sensitive optical microscopy relying on local reflectance changes at an absorbing material (here $\mathrm{Au}$ ), was used to image and size dynamically and in situ dielectric and metallic NPs. We show how BALM images can quantify the local electrodeposition or stripping of $\mathrm{Ag}$ nanomaterial onto a BALM electrode to the point of producing single NP voltammograms associated to these processes down to $10 \mathrm{~nm}$ NP. The controlled electrodeposition process produces arrays of Ag NPs of wide size distribution. Such platform, through BALM monitoring, provides new insight into nanoscale electrochemistry. Comparing the electrochemical and optical response indirectly supports the contribution of nanoclusters aggregation during 3D NP electrodeposition. Then the NP arrays were used to analyze in the same experiments size-dependent electrochemistry at the single NP level. We show that size effect has different origins: the NP nucleation probability for the electrodeposition potential and a surface energy contribution for the electrodissolution potential. In the latter case, we confirm, at the single NP level, recent reports showing that surface energy contributions are often overestimated. Finally, the method was extended to more general chemical NP transformation exemplified here in the in situ visualization and quantification of electrochemical Ostwald ripening.

\section{Acknowledgements}

We are grateful for financial support by the Agence Nationale pour la Recherche (NEOCASTIP-ANR-15-CE09-0015-02).

Keywords: electrodeposition $\cdot$ optical microscopy $\cdot$ single nanoparticle electrochemistry $\bullet$ size effects

\section{References}

[1] C. Costentin, C. di Giovanni, M. Giraud, J.M. Savéant, C. Tard, C. Nat. Mat. 2017, 16, 1016-1022.

[2] a) S.E.F. Klein, S.C.S. Lai, T.M. Koper, P.R. Unwin, Angew. Chem. Int. Ed. 2014, 53, 3558-3586; b) R.M. Penner, J. Phys. Chem. B 2002, 106, 3339-3353.

[3] a) W. Wang, Chem. Soc. Rev. 2018, 47, 2485-2508; b) Y. Wang, X Shan, N.; Tao, Faraday Discuss. 2016, 193, 9-39; c) C.M. Hill, S. Pan J. Am. Chem. Soc. 2013, 135, 17250-17253; d) Y. Fang, W. Wang, X Wo, Y. Luo, S. Yin, Y. Wang, X. Shan, N. Tao, N. J. Am. Chem. Soc. 2014, 136, 12584-12587; e) V. Brasiliense, A.N. Patel, A. Martinez Marrades, J. Shi, Y. Chen, C. Combellas, G. Tessier, F. Kanoufi, J. Am Chem. Soc. 2016, 138, 3478-3483; f) J. Smith, Q. Yang, P.K. Jain Angew. Chem. Int. Ed. 2014, 53, 2967-2872.

[4] a) S. Campidelli, R.A. Khachfe, K. Jaouen, J. Monteiller, C. Amra, M. Zerrad, R. Cornut, V. Derycke, D. Ausserré, Sci. Adv. 2017, 3 , e1601724; b) http://www.watchlive.fr/index.php/en/ Accessed June 13, 2018

[5] a) J.V. Zoval, R.M. Stiger, P.R. Biernacki, R.M. Penner, J. Phys. Chem. 1996, 100, 837-844; b) A. Radisic, P.M. Vereecken, J. B. Hannon, P.C Searson, F.M. Ross, NanoLett. 2006, 6, 238-242; c) J. Ustarroz, J.A Hammons, T. Atlantzis, A. Hubin, S. Bals, H. Terryn, J. Am. Chem. Soc. 2013, 135, 11550-11561; d) R L. Harniman, D. Plana, G.H. Carter, K.A. Bradley, M.J. Miles, D.J. Fermin, Nat. Comm. 2017, 8, 971; e) S.C.S Lai, R.A. Lazenby, P.M. Kirkman, P.R. Unwin, Chem. Sci. 2015, 6, 1126-1138.

[6] M.E. Hyde, R.G. Compton, J. Electroanal. Chem. 2003, 549, 1-12.

[7] J. Kim, J.E. Dick, A.J. Bard, Acc. Chem. Res. 2016, 49, 2587-2595.

[8] G.G. Daaboul, A. Yurt, X. Zhang, G.M. Hwang, B.B. Goldberg, M.S. Ünlü, NanoLett. 2010, 10, 4727-4731.

[9] a) S. Wang, X. Shan, U. Patel, X. Huang, J. Lu, J. Li, N. Tao, Proc. Nat Acad. Sci. 2010, 107, 16028-16032; b) S. Munteanu, J. P. Roger, Y Fedala, F. Amiot, C. Combellas, G. Tessier, F. Kanoufi, Faraday Discuss. 2013, 164, 241-258; c) S. Munteanu, N. Garraud, J. P. Roger F. Amiot, J. Shi, Y. Chen, C. Combellas, F. Kanoufi, Anal. Chem. 2013 85, 1965-1971.

[10] K. Ngamchuea, R.O.D. Clark, S. V. Sokolov, N.P. Young, C. Batchelor McAuley, R.G. Compton, Chem. A Eur. J. 2017, 23, 16085-16096.

[11] A. Milchev, J. Electroanal. Chem. 1998, 457, 35-46.

[12] W.J. Plieth, J. Phys. Chem. 1982, 86, 3166-3170.

[13] a) C.C. M. Neumann, C. Batchelor-McAuley, K. Tschulik, H.S. Toh, P Shumbula, J. Pillay, R. Tshikhudo, R.G. Compton, ChemElectroChem 2014, 1, 87-89; b) O.S. Ivanova, F.P. Zamborini, J. Am. Chem. Soc 2010, 132, 70-72.

[14] H.S. Toh, C. Batchelor-McAuley, K. Tschulik, M. Uhlemann, A. Crossley, R.G. Compton, Nanoscale 2013, 5, 4884-4893.

[15] M. Hasheminejad, Y. Fang, M. Li, Y. Jiang, W. Wang, H.Y. Chen, Angew. Chem., Int. Ed. 2017, 56, 1629-1633. 
Layout 1:

\section{COMMUNICATION}

Disordered arrays of wide size distribution of $\mathrm{Ag}$ nanoparticles are generated by progressive electrodeposition. Combined to highly sensitive optical microscopy such platform grants access to sizedependent single nanoparticle electrochemistry.

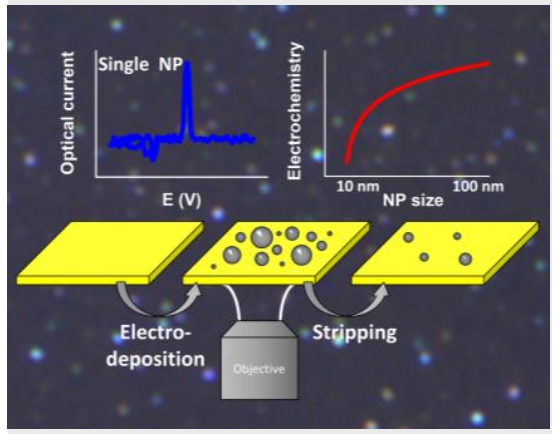

J.-F. Lemineur, J.-M. Noël, D. Ausserré, C. Combellas, F. Kanoufi*

Page No. - Page No.

Combining electrodeposition and optical microscopy for probing sizedependent single nanoparticle electrochemistry 